Editorial

\title{
Acknowledgement to Reviewers of Toxics in 2016
}

\section{Toxics Editorial Office}

Published: 11 January 2017

MDPI AG, St. Alban-Anlage 66, 4052 Basel, Switzerland; toxics@mdpi.com

The editors of Toxics would like to express their sincere gratitude to the following reviewers for assessing manuscripts in 2016.

We greatly appreciate the contribution of expert reviewers, which is crucial to the journal's editorial process. We aim to recognize reviewer contributions through several mechanisms, of which the annual publication of reviewer names is one. Reviewers receive a voucher entitling them to a discount on their next MDPI publication and can download a certificate of recognition directly from our submission system. Of course, in these initiatives we are careful not to compromise reviewer confidentiality. Many reviewers see their work as a voluntary and often unseen part of their role as researchers. We are grateful to the time reviewers donate to our journals and the contribution they make.

If you are interested in becoming a reviewer for Toxics, see the link at the bottom of the webpage http://www.mdpi.com/reviewers.

The following reviewed for Toxics in 2016:

$\begin{array}{lll}\text { Anadon, Arturo } & \text { D'annibale, Alessandro } & \text { Lin, Zhoumeng } \\ \text { Antunes, Margarida } & \text { De Gennaro, Gianluigi } & \text { Liu, Hui-Ming } \\ \text { Albuquerque, Teresa } & \text { De Lourdes Pereira, Maria } & \text { Liu, Shing-Hwa } \\ \text { Alexander, Laura E. Crotty } & \text { De Oliveira Fernandes, Eduardo } & \text { Looger, Loren } \\ \text { Aris, Aziz } & \text { Devillers, James } & \text { Luzio, Ana } \\ \text { Bailey, Helen } & \text { DeWitt, Jamie C. } & \text { Mackinnon, Gillian } \\ \text { Barrett, Emily S. } & \text { Drapeau, Pierre } & \text { Maricle, Brian R. } \\ \text { Bäumler, Hans } & \text { Evelyn, Talbott } & \text { Myridakis, Antonis } \\ \text { Baxter, Lisa } & \text { Faggio, Caterina } & \text { Nasuti, Cinzia } \\ \text { Bellinger, David } & \text { Finazzi, Francesco } & \text { Oh, Jin-Woo } \\ \text { Blukacz-Richards, E. Agnes } & \text { Fitsanakis, Vanessa } & \text { Orford, Rob } \\ \text { Bodin, Johanna } & \text { Fletcher, John S. } & \text { Padilla, Stephanie } \\ \text { Boogaard, Peter } & \text { Fujihara, Junko } & \text { Peyton, David H. } \\ \text { Bouchard, Michèle } & \text { Gangula, Pandu } & \text { Pivato, Alberto } \\ \text { Brown, Grant E. } & \text { Georgieva, Radostina } & \text { Porsch Hällström, Inger } \\ \text { Bury, Nic R. } & \text { Greim, Helmut } & \text { Pottinger, Tom G. } \\ \text { Chai, Tianfeng } & \text { Harrison, Roy } & \text { Powell, Craig } \\ \text { Chan, King Ming } & \text { Hatch, Elizabeth } & \text { Radbruch, Lucas } \\ \text { Chen, Jiangang } & \text { Hendryx, Michael } & \text { Rainieri, Sandra } \\ \text { Chen, Te-Hao } & \text { Hosgood, Howard Dean } & \text { Ramesh, Tennore M. } \\ \text { Cheng, Delfine } & \text { Kim, Yong Pyo } & \text { Rogiers, Bart } \\ \text { Chrzanowski, Łukasz } & \text { Koehler, Raymond } & \text { Sacks, Jason D. } \\ \text { Churchill, David G. } & \text { Kojima, Hiroyuki } & \text { Sarras, Michael P. Jr. } \\ \text { Cicero, Theodore J. } & \text { Kwak, Wan-Sup } & \text { Saunders, David M.V. } \\ \text { Conti, Barbara } & \text { Lachenmeier, Dirk } & \text { Schnug, Ewald } \\ \text { Crunelle, Cleo } & \text { Lai, Chia-Hsiang } & \text { Sekine, Yoshika } \\ \text { Cyr, Daniel G. } & \text { Lee, Jin Heon } & \text { Semple, Sean } \\ & & \\ & & \end{array}$


Setou, Mitsutoshi

Silva, Lígia

Simon, Eszter

Snow, Mathew S.

Sogorb, Miguel A.

Sone, Hideko
Tava, Aldo

Tigini, Valeria

Touboul, David

Ulrich, Franck

Wallace, David

Walton, AnnMarie

\author{
Wang, Wei \\ Ward, Tony \\ Worek, Franz \\ $\mathrm{Xu}$, Xiaohui
}

(c)

(C) 2017 by the author; licensee MDPI, Basel, Switzerland. This article is an open access article distributed under the terms and conditions of the Creative Commons Attribution (CC-BY) license (http://creativecommons.org/licenses/by/4.0/). 